\title{
The QED Structure of the Photon
}

\author{
Richard Nisius, on behalf of the LEP Collaborations] \\ CERN, CH-1211 Genève 23, Switzerland
}

\begin{abstract}
Measurements of the QED structure of the photon based on the reaction ee $\rightarrow \operatorname{ee} \gamma^{(\star)}\left(P^{2}\right) \gamma^{\star}\left(Q^{2}\right) \rightarrow$ ee $\mu \mu$ are discussed. This review is an update of the discussion of the results on the QED structure of the photon presented in Refs. [1], and covers the published measurements of the photon structure functions $F_{2}^{\gamma}, F_{\mathrm{A}}^{\gamma}$ and $F_{\mathrm{B}}^{\gamma}$ and of the differential cross-section $\mathrm{d} \sigma / \mathrm{d} x$ for the exchange of two virtual photons.
\end{abstract}

\section{Introduction}

Several measurements concerning the QED structure of the photon have been performed by various experiments. Prior to LEP, mainly the structure function $F_{2}^{\gamma}$ of the quasi-real photon, $\gamma$, as a function of the photon virtuality $Q^{2}$ of the virtual photon, $\gamma^{\star}$, was measured. The LEP experiments refined the analysis of the $\mu \mu$ final state, and derived more information on the QED structure of the photon. The interest in the investigation of the QED structure of the photon is twofold. Firstly the investigations serve as tests of QED to $\mathcal{O}\left(\alpha^{4}\right)$, but secondly, and also very important, the investigations are used to refine the experimentalists tools in a real but clean experimental environment to investigate the possibilities of extracting similar information from the much more complex hadronic final state.

\section{The photon structure function $F_{2}^{\gamma}$}

The structure function $F_{2}^{\gamma}$ has been measured using data in the $Q^{2}$ range from about 0.14 up to $400 \mathrm{GeV}^{2}$. Results were published by the CELLO [2], DELPHI [3, L3 [4], OPAL [5], PLUTO [6] and TPC $/ 2 \gamma$ [7] experiments. Special care has to be taken when comparing the experimental results to the QED predictions, because slightly different quantities are derived by the experiments. Some of the experiments express their result as an average structure function, $\left\langle F_{2}^{\gamma}\left(x, Q^{2}\right)\right\rangle$, measured within their experimental acceptance in $Q^{2}$, whereas the other experiments unfold their result as a structure function for an average $Q^{2}$ value, $F_{2}^{\gamma}\left(x,\left\langle Q^{2}\right\rangle\right)$. Figure 1 shows the world summary of the $F_{2}^{\gamma}$ measurements compared either to $\left\langle F_{2}^{\gamma}\left(x, Q^{2}\right)\right\rangle$, assuming a flat acceptance in $Q^{2}$, or

\footnotetext{
${ }^{1}$ Invited talk given at the 7th International Workshop on Deep Inelastic Scattering and QCD, April 19 to 23, 1999, Zeuthen, to appear in the proceedings
} 
to $F_{2}^{\gamma}\left(x,\left\langle Q^{2}\right\rangle\right)$, using the appropriate values for $Q^{2}$ and $\left\langle Q^{2}\right\rangle$ given by the experiments. For the measurements which quote an average virtuality $\left\langle P^{2}\right\rangle$ of the quasi-real photon for their dataset, this value is chosen in the comparison, otherwise $P^{2}=0$ is used. There is a nice agreement between the data and the QED expectations for about three orders of magnitude in $Q^{2}$. The LEP data are so precise that the effect of the small virtuality of the quasi-real photon can clearly be established, as shown, for example, in Figure 2 for the most precise data from OPAL. The data are compared to the QED predictions of $F_{2}^{\gamma}\left(\mathrm{x},\left\langle Q^{2}\right\rangle,\left\langle P^{2}\right\rangle, m_{\mu}\right)$, where either $\left\langle P^{2}\right\rangle$ or $m_{\mu}$ is varied. Using this data, the mass of the muon is found to be $m_{\mu}=0.113_{-0.017}^{+0.014} \mathrm{GeV}$, assuming the $\left\langle P^{2}\right\rangle$ value predicted by $\mathrm{QED}$. Although this is not a very precise measurement of the mass of the muon it can serve as an indication on the precision possible for the determination of $\Lambda$, if it only were for the pointlike contribution to $F_{2, \text { had }}^{\gamma}$.

\section{Azimuthal correlations}

The structure functions $F_{\mathrm{A}}^{\gamma}$ and $F_{\mathrm{B}}^{\gamma}$ are obtained from the measured $F_{2}^{\gamma}$, and a fit to the shape of the distribution of the azimuthal angle $\chi$, which is the angle between the plane defined by the momentum vectors of the muon pair and the plane defined by the momentum vectors of the incoming and the deeply inelastically scattered electron. For small values of $y$, the $\chi$ distribution can be written as:

$$
\frac{\mathrm{d} N}{\mathrm{~d} \chi} \sim 1-F_{\mathrm{A}}^{\gamma} / F_{2}^{\gamma} \cos \chi+\frac{1}{2} F_{\mathrm{B}}^{\gamma} / F_{2}^{\gamma} \cos 2 \chi .
$$

The recent theoretical predictions from Ref. [8] which take into account the important mass corrections up to $\mathcal{O}\left(m_{\mu}^{2} / W^{2}\right)$, are consistent with the measurements of Refs. [4, 5], Figure 3. Both $F_{\mathrm{A}}^{\gamma}$ and $F_{\mathrm{B}}^{\gamma}$ are found to be significantly different from zero. The shape of $F_{\mathrm{B}}^{\gamma}$ cannot be determined very accurately, but it is not compatible with a constant. The best fit to a constant $F_{\mathrm{B}}^{\gamma} / \alpha$ leads to 0.032 and 0.042 with $\chi^{2} /$ dof of 8.9 and 3.1 for the L3 and OPAL results respectively.

\section{Cross-section for highly virtual photons}

The cross-section for the exchange of two highly virtual photons in the kinematical region under study can schematically be written as:

$$
\begin{aligned}
\sigma \sim & \sigma_{\mathrm{TT}}+\sigma_{\mathrm{TL}}+\sigma_{\mathrm{LT}}+\sigma_{\mathrm{LL}}+ \\
& \frac{1}{2} \tau_{\mathrm{TT}} \cos 2 \bar{\phi}-4 \tau_{\mathrm{TL}} \cos \bar{\phi} .
\end{aligned}
$$

Here the total cross-sections $\sigma_{\mathrm{TT}}, \sigma_{\mathrm{TL}}, \sigma_{\mathrm{LT}}$ and $\sigma_{\mathrm{LL}}$ and the interference terms $\tau_{\mathrm{TT}}$ and $\tau_{\mathrm{TL}}$ correspond to specific helicity states of the photons $(\mathrm{T}=$ transverse 
and $\mathrm{L}=$ longitudinal), and $\bar{\phi}$ is the angle between the electron scattering planes. There is good agreement between $\mathrm{d} \sigma / \mathrm{d} x$ measured by OPAL, Figure 4 , and the QED predictions using the Vermaseren [9] and the GALUGA [10] Monte Carlo programs, provided all terms of the differential cross-section are taken into account. However, as apparent from Figure 1, if either $\tau_{\mathrm{TT}}$ (dot-dash) or both $\tau_{\mathrm{TT}}$ and $\tau_{\mathrm{TL}}$ (dash) are neglected in the QED prediction as implemented in the GALUGA Monte Carlo, there is a clear disagreement between the data and the QED prediction. This measurement clearly shows that both terms, $\tau_{\mathrm{TT}}$ and especially $\tau_{\mathrm{TL}}$, are present in the data in the kinematical region of the OPAL analysis. The contributions to the cross-section are negative and large, mainly at $x>0.1$.

As the kinematically accessible range in terms of $Q^{2}$ and $P^{2}$ for the measurement of the QED and the QCD structure of the photon is the same, and given the size of the interference terms in the leptonic case, special care has to be taken when the measurements of the QCD structure are interpreted in terms of hadronic structure functions of virtual photons.

\section{Conclusions}

QED has been tested to $\mathcal{O}\left(\alpha^{4}\right)$ using the reaction ee $\rightarrow$ ee $\gamma^{(\star)} \gamma^{\star} \rightarrow$ ee $\mu \mu$, and was found to be in good agreement with all experimental results. Because the precision of the measurements is limited mainly by the statistical error, significant improvements will be made by using the full expected statistics of $500 \mathrm{pb}^{-1}$ of the LEP2 programme.

Acknowledgement:

I wish to thank the organisers of this interesting workshop for the fruitful atmosphere they created throughout the meeting.

\section{References}

[1] R. Nisius, HEP97 Conf., Jerusalem, 1997, hep-ex/9712012; R. Nisius, DIS98 Conf., Brussels, 1998, edited by G. Coremans and R. Roosen, p194198, World Scientific, 1998.

[2] CELLO Collab., H.J. Behrend et al., Phys. Lett. 126B, 384-390 (1983).

[3] DELPHI Collab., P. Abreu et al., Z. Phys. C69, 223-234 (1996).

[4] L3 Collab., M. Acciarri et al., Phys. Lett. B438, 363-378 (1998).

[5] OPAL Collab., G. Abbiendi et al., CERN-EP/99-010, subm. Eur. Phys. J. C.

[6] PLUTO Collab., C. Berger et al., Z. Phys. C27, 249-256 (1985). 
[7] TPC/2 $\gamma$ Collab., M.P. Cain et al., Phys. Lett. 147B, 232-236 (1984).

[8] R. Nisius and M.H. Seymour, Phys. Lett. B452, 409-413 (1999).

[9] J.A.M. Vermaseren, Nucl. Phys. B229, 347-371 (1983), and Refs. therein.

[10] G.A. Schuler, Comp. Phys. Comm. 108, 279-303 (1998). 

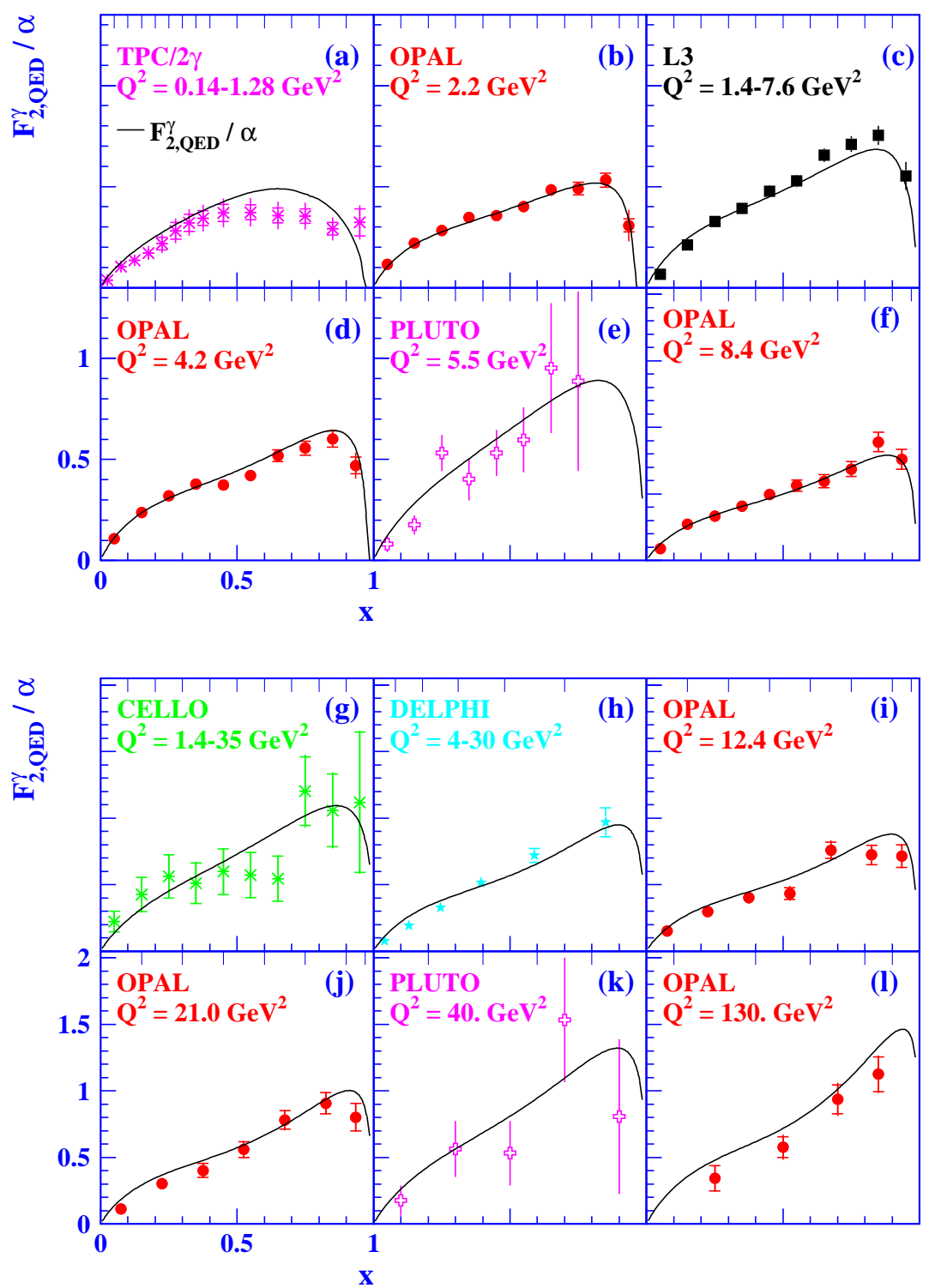

Figure 1: The world summary on $F_{2}^{\gamma}$ measurements. The quoted errors for $(\mathrm{h})$ are statistical only. 

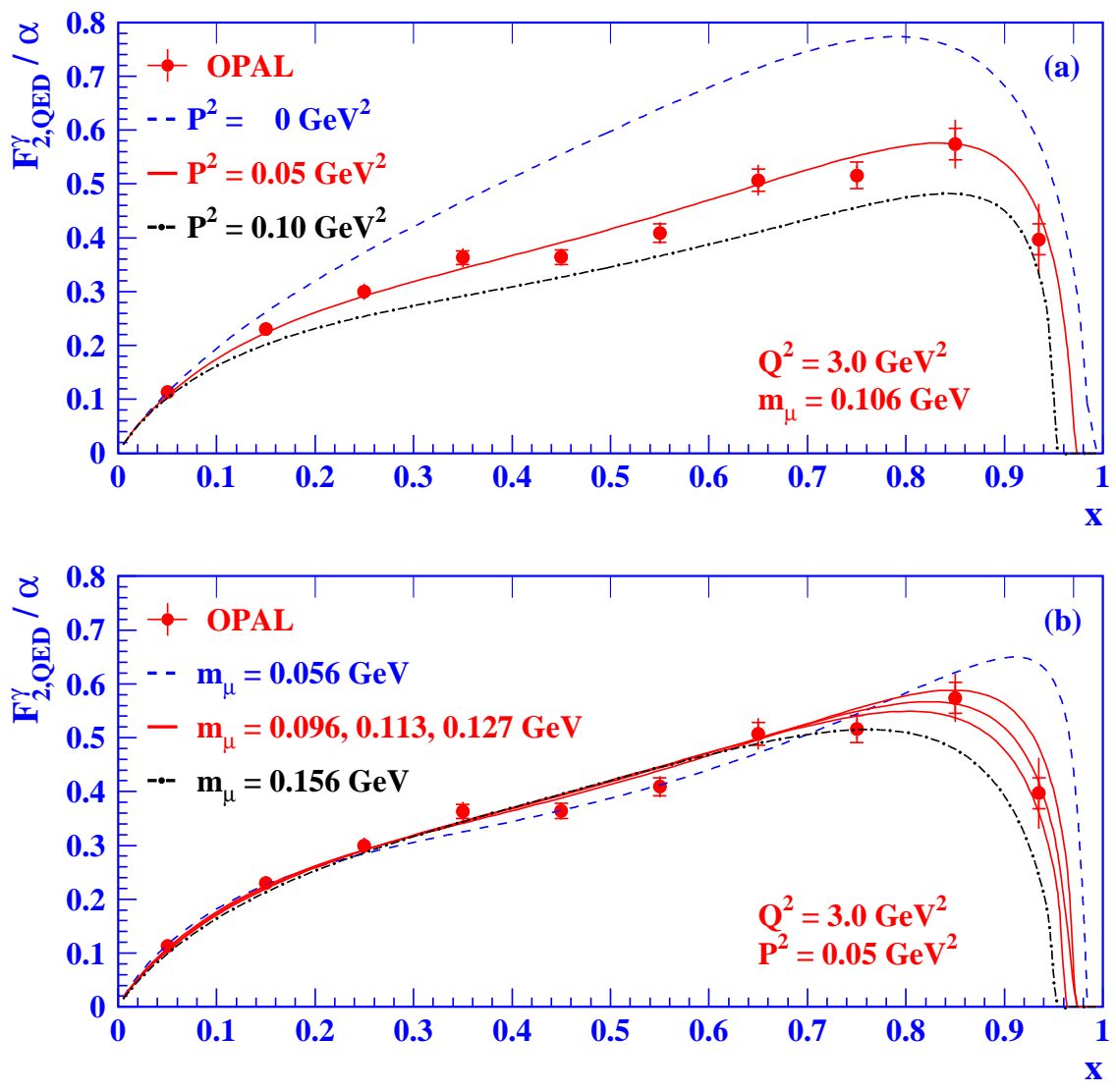

Figure 2: The dependence of $F_{2}^{\gamma}$ on $P^{2}$ and on the mass of the muon, $m_{\mu}$. 

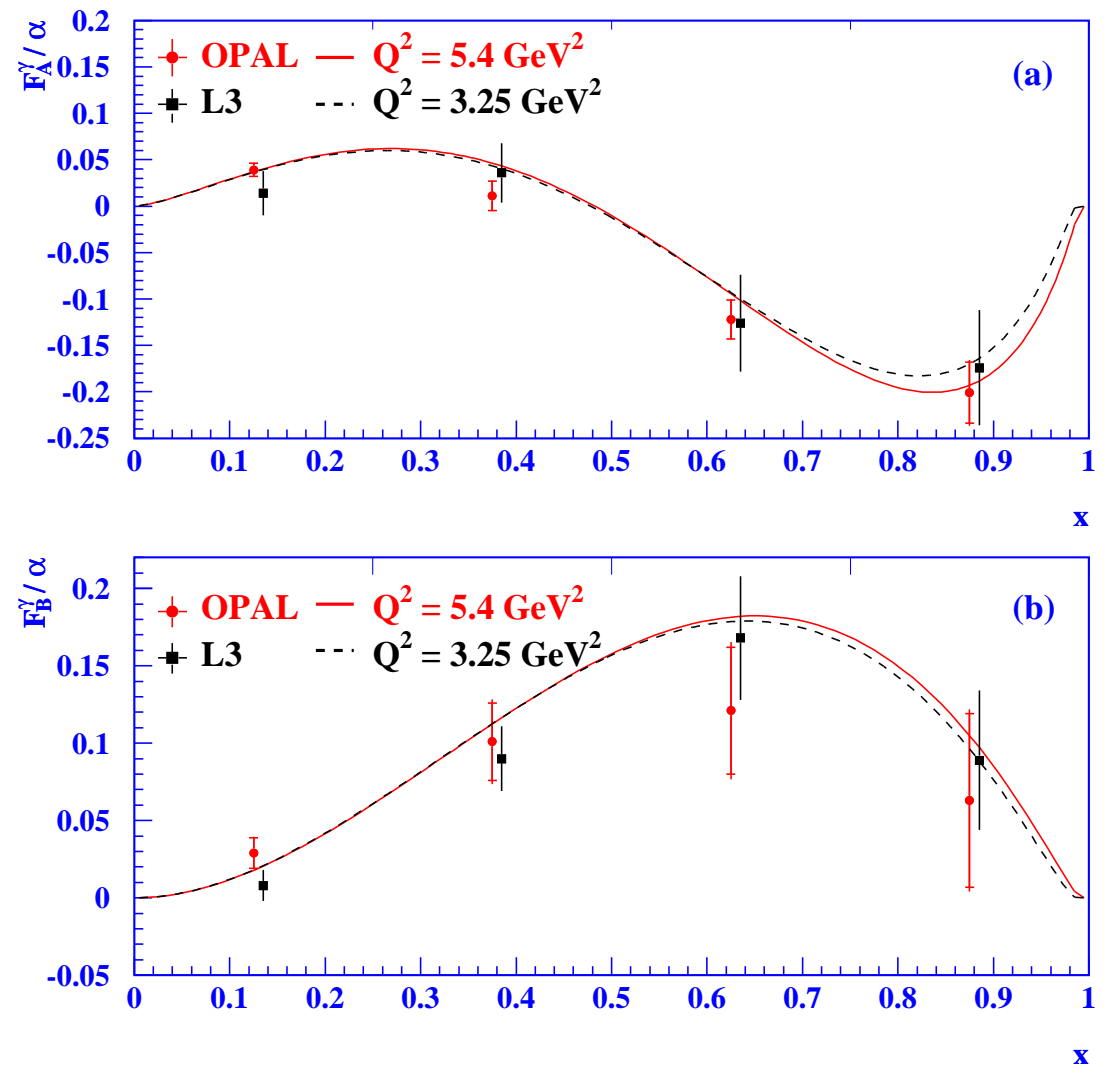

Figure 3: The measurements of $F_{\mathrm{A}}^{\gamma}$ and $F_{\mathrm{B}}^{\gamma}$ from L3 and OPAL. 

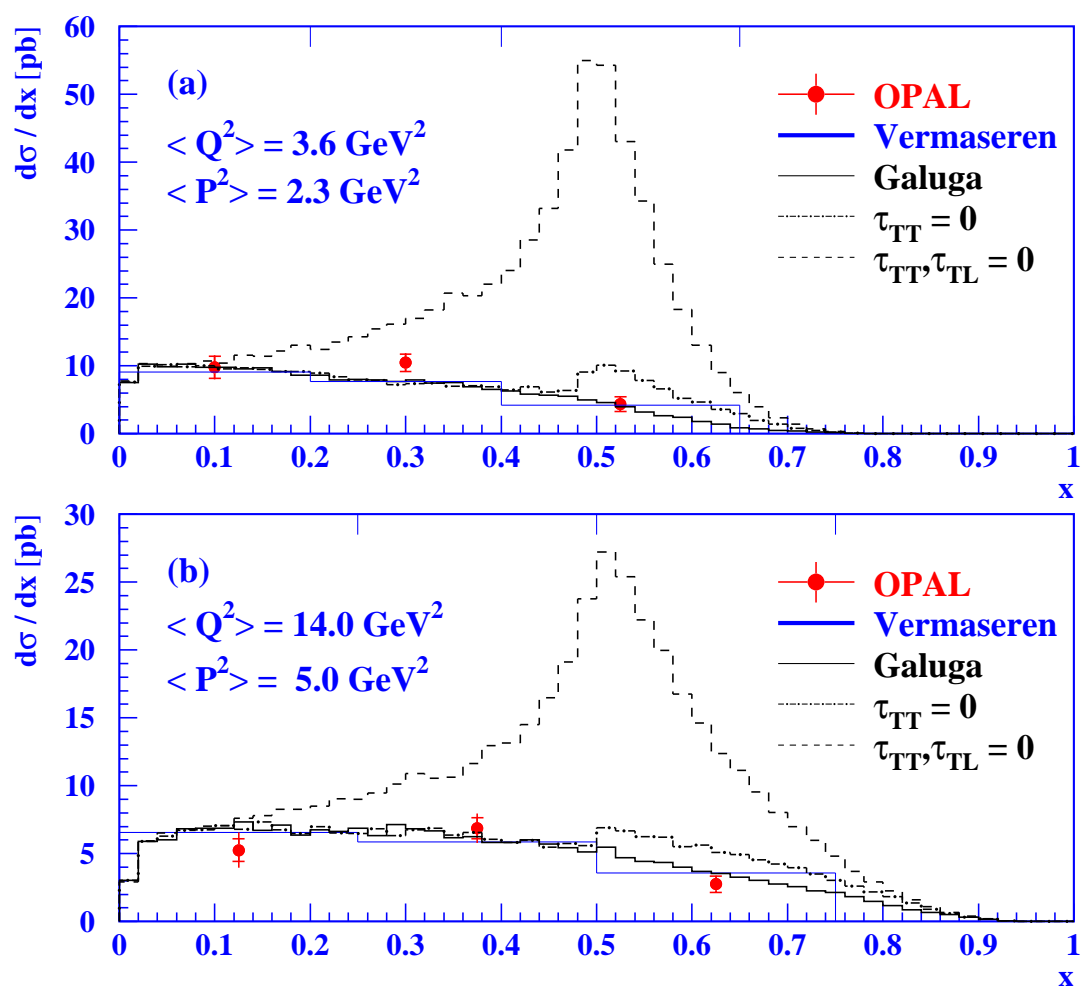

Figure 4: The measurement of the differential cross section $\mathrm{d} \sigma / \mathrm{d} x$ for highly virtual photons from OPAL. 